\title{
Photobiomodulation therapy combined to static magnetic field before blood flow restriction training in untrained individuals: protocol for a double-blind, randomized, placebo-controlled trial
}

\author{
João Vitor Ferlito ${ }^{*}$; Ernesto Cesar Pinto Leal-Junior²; Mirian Salvador ${ }^{1}$; Thiago De Marchi ${ }^{1,2}$. \\ ${ }^{1}$ Laboratório de Estresse Oxidativo e Antioxidantes (LEOA), Universidade de Caxias do Sul (UCS), Caxias do Sul, RS, Brasil; ${ }^{2}$ Laboratório de \\ Fototerapia e Tecnologias Inovadoras e Saúde (LaPIT), Universidade Nove de Juho (UNINOVE), São Paulo, SP, Brasil.
}

\section{ABSTRACT}

Background: Photobiomodulation therapy with static magnetic field (PBMT/sMF) stands out for being a non-pharmacological resource with bioenergetic effects capable of accelerating muscle recovery, delaying muscle fatigue and potentiate gains in different training protocols. In recent years, blood flow restriction (BFR) associated with low load exercise (20-30\% 1RM/MVC) has demonstrated positive effects on muscle performance. However, the effects of PBMT/sMF combined with BFR training are still unknown. Objective: Verify the effects of PBMT/sMF associated with BFR training and compared with high load training (HLT) in the muscle strength, muscle damage, inflammation, and the oxidative stress. Methods: This are a protocol of a randomized, double-blind, placebo-controlled clinical trial. The participants will be healthy men between the ages of 18 to 40 years, with no practice in upper limb strength training in the previous three months. The voluntaries will be randomly divided into four groups: (1) PBMT/sMF + BFR; (2) PBMT/sMF + HLT; (3) placebo + BFR; (4) placebo + HLT. The PBMT/sMF will be applied immediately before strengthening protocol (4 sets $\times 20$ repetitions of elbow flexion). The BFR groups will undergo to exercise with low load (30\% of MVC), while the HLT groups will performed the same protocol with $80 \%$ of MVC. The primary outcome will be muscle strength, measured in baseline, fourth, eighth week of training and detraining period. The secondary outcomes include measured the fatigue resistance, arm circumference, muscle damage, inflammatory and oxidative stress levels in one session, during, after intervention and detraining. Discuss: This trial will elucidate the effects of PBMT/sMF when when used in association with BFR training or HLT.

Keywords: Phototherapy; Muscle performance; Occlusion vascular; Oxidative stress; Exercise.

\section{BACKGROUND}

The resistance exercise $(\mathrm{RE})$ is an important method used for prevention, rehabilitation, maintenance of health, and quality of life, increasing practitioners' number, regardless of age or sex..$^{(1,2)}$ These individuals aim to achieve through RE benefits such as increased strength, muscle mass, and sports performance. ${ }^{(3)} \mathrm{RE}$ with high load training (HLT) appears as the most effective method for developing muscle strength, and, since the last two decades it has been a field of increasing research due your beneficial effects in health human. It is known, the RE entail a systemic change in the body through physiological adaptations in anaerobic and aerobic metabolism, changing the neuromuscular system, increasing muscles fibers numbers, and inducing muscle hypertrophy. ${ }^{(2-4)}$

The American College Sports Medicine (ACSM) and other authors recommend that in order to achieve significant muscle strength gain and hypertrophy, a load greater than $70 \%$ of a maximum repetition (1RM) or maximal voluntary contraction (MVC) should be used. ${ }^{(5,6)}$ Unlike several types of RE that used high intensity $(70 \%-85 \%)$, blood flow restriction (BFR) training with low load (20-50\% 1RM or MVC), called Kaatsu training, stands out. (7) This training method showed positive results, as increased muscle strength and mass, similar to high-load training (HLT), so much in adults, ${ }^{(8,9)}$ elderly, ${ }^{(10-12)}$ and athletes. $(13,14)$

One of RE's effects due to intensity use and repetitive of musculature is muscle fatigue, being responsible for a progressive decline in performance. Muscle fatigue also contributes to muscle damage, which corresponds to an inflammatory response, with an increase in blood muscle proteins and delayed onset muscle soreness. (15-17) Due to the growing scientific interest in the search for resistance in exercise, scientists have been studying ways to measure muscle damage induced by exercise. Therefore, measurements of the activity of the enzyme creatine kinase (CK) have been used to determine muscle damage and recovery process. ${ }^{(17,18)}$

Some authors pinpoint that the BFR training possibly induces fatigue earlier than HLT due to your metabolic stress, caused by local hypoxic on muscle (19). Metabolic stress is currently known as a physiological process that occurs during exercise in response to low energy that leads to metabolite accumulation [lactate, phosphate inorganic $(\mathrm{Pi})$, and ions of hydrogen $(\mathrm{H}+)$ ] in muscle cells. ${ }^{(9,20)}$ The metabolic stress can produce Oxigen-reactive species (ROS), which at moderate antioxidant capacity and immune response ${ }^{(21)}$. 
Recent studies showed that BFR effects had shown an increase in the cytokine IL-6, which plays a and skeletal muscle remodeling ${ }^{(20)}$. This associated response is associated with the hypoxic muscle environment generated by BFR and ROS production, contributing to the increased demand for energy production by anaerobic metabolism and glycogen depletion ${ }^{(12,20)}$. However, studies investigating whether BFR can modulate acutely, and chronically the antioxidant and inflammatory responses present controversial results. In addition, lack of detailed information on BFR application, exercise protocol, small sample size and data loss affect the reliability of the results and result in a high risk of bias. The scarcity of clinical trials with reliable results and high methodological quality demonstrates the need for further studies on the topic to elucidate the effects of BFR on oxidative stress and ROS production.

More recently, Vanin et al (22) also shown that PBMT/sMF can potentiate gains in different training protocols, such as strength training. The PBMT is defined as a light therapy that uses nonionizing light sources, such as lasers, lightemitting diodes (LEDs), and broadband light, from the visible to the infrared spectrum. ${ }^{(23)}$ Recent evidences have shown that PBMT can positively affect the antioxidant activity and improve musculoskeletal conditions ${ }^{(24-27)}$. In addition, PBMT has been used in combination with a static magnetic field (sMF) (28), generating better effects on cell metabolism. ${ }^{(29)}$ Although PBMT or $\mathrm{PBMT} / \mathrm{sMF}$ has considerably increased the number of searches in recent years, most studies have evaluated only PBMT/ sMF acute effects (27). However, the effects of PBMT/sMF therapy combined with BFR training on muscle performance and biochemical responses have not yet been investigated.

The purpose of this study is to evaluate the effects of PBMT/sMF with BFR training in muscle performance. Thus, this clinical trial will test the hypothesis that the combination of PBMT/ sMF with BFR may be an approach capable of providing adaptations of the skeletal musculature, the redox system, and the inflammatory response, with a consequent increase in muscle performance.

\section{METHODS}

This is a prospectively registered, four-arm randomized placebo-controlled trial with concealed allocation, blinded measurers and volunteers, intention-to-treat analysis, and one month of follow-up (detraining period). All the study components will be completed at the Sports Medicine Institute (SMI). The study follows the SPIRIT (Standard Protocol Items: Recommendations for International Trials) 2013 checklist ${ }^{(30)}$ (Additional file 1) and the TIDieR (Template for Intervention Description and Replication) ${ }^{(31)}$.

\section{Ethical aspects}

The study was approved by the Ethics Committee of the University Center UNICNEC. Following the Declaration of Helsinki, all volunteers were advised about the procedure and signed informed consent before participation in the study (CAEE 09865119.7.000.5571). This trial is registered in the Register of Brazilian Clinical Trials (REBEC), number RBR-3623xj. The volunteers will then be informed about the procedures and will sign a statement of informed consent in compliance with Resolution 196/96 of the Brazilian National Board of Health before the execution of the study. All personal data will be confidential.

\section{Participants}

For recruitment of volunteers, initial contact will be made through social media, afterward, the volunteers will be solicited to appear at Institute of Sports Medicine (IME), the research team will ask about their personal data to identify if they meet the inclusion criteria or if they need to be excluded from the clinical trial. A questionnaire will be administered addressing age (years), body mass (kilograms), height (centimeters), not dominant upper limb, schooling (no schooling, elementary school education, high school education, university education, or postgraduate degree), and marital status (single, married or widowed).

\section{Eligibility Criteria}

Pre-defined inclusion criteria will be employed (1) healthy individuals, (2) male, (3) age between 18 - 40 years, (4) do not be realizing resistance training in the previous three months. The voluntaries with the following characteristics will be excluded from the study, (1) history of musculoskeletal injury to the upper limb, (2) history of surgical, (3) history of cardiovascular pathology, (4) history of venous thrombosis and (5) use of pharmacological agents or nutritional supplements. Subjects who discontinue participation in the study will be invited to participate in the assessment three months after 
the start of treatment (week twelfth of the study). Thus, all individuals will be included in the intention-to-treat analysis. Adherence to treatment will be assessed by calculating the percentage of sessions completed by each volunteer. Patients will be instructed not to drink alcohol or food before training sessions. If the patient has a fever or malaise session, he will be asked to reschedule the session.

\section{Randomization and Blinding}

Seeking to minimize selection bias and ensure that all voluntaries will be randomly allocated to any group, the block randomization will be performed by a researcher who will not have contact with the study subjects or other researchers involved in the project.

Another researcher will be responsible for programming the device (PBMT/sMF or placebo) and will be instructed not to inform volunteers or other researchers about the type of treatment (PBMT/sMF or placebo). The sounds and signals emitted by the device, as well as the information displayed on the screen, were identical, regardless of the type of treatment (PBMT//sMF or placebo), providing adequate concealment by volunteers and therapists. All volunteers will be used a blindfold during treatments, both for safety and to help blind them. Thus, volunteers and evaluators were blinded to maintain the doubleblind design. Randomization tags will be created at www.randomization.com, and a series of sealed, opaque, and numbered envelopes will be used to ensure confidentiality and to determine which experimental group each volunteer will be allocated. Volunteers will be allocated as reported below:

(1) $\mathrm{PBMT} / \mathrm{sMF}+\mathrm{BFR}: \mathrm{PBMT} / \mathrm{sMF}$ before the BFR training sessions;

(2) PBMT/sMF + HLT: PBMT/sMF before the HLT sessions;

(3) placebo + BFR: placebo before the BFR training sessions;

(4) placebo + HLT: placebo before the HLT training sessions.

The evaluations will be performed before starting the protocol (baseline) and after four, eight weeks of sessions training, and one month of follow-up (detraining period). The blood samples and biochemical analyses evaluation will happen in the first training session (1 $\mathrm{min}$ - before and after) in all groups to verify the acute effects. A flow chart that summarizes the procedures that will be performed in this study is shown in Figure 1. Study design schedule in accordance with the SPIRIT checklist is showed in Painting 1.

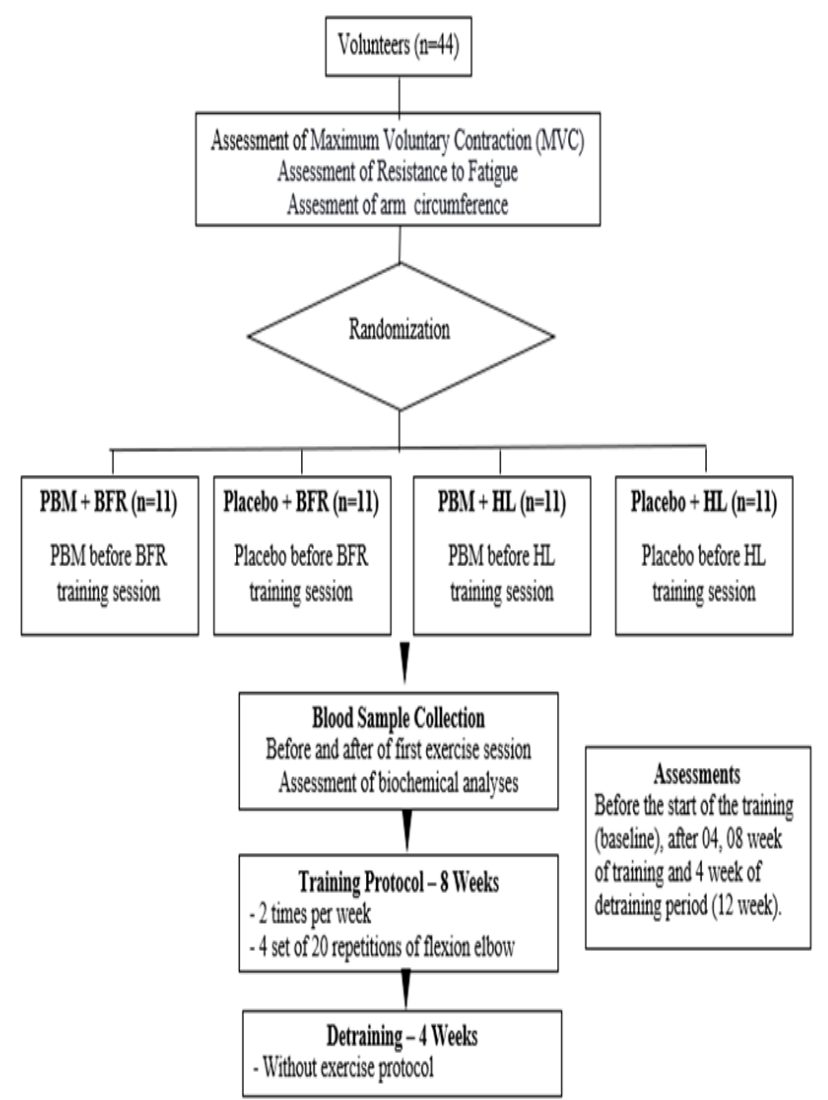

Figure 1. Flow diagram

Note: BFR, blood flow restriction, PBM, photobiomodulation therapy, $\mathrm{HL}=$ high load.

\section{Interventions}

\section{Training session}

Training sessions will be held at a 48-hour interval between each session. Upon arrival the voluntaries will be submitted to a warm-up period of $5 \mathrm{~min}$ the ergometer cycle (Acte Sports, São Paulo, Brazil). Later the voluntaries will be led and actively submitted to PBMT/ sMF or placebo in both upper limbs. After this, the subject will perform the training protocol focused on the biceps brachii muscle. All volunteers will be instructed not to eat any food one hour before training, alcoholic drink and caffeine. The adverse events will be monitoring during all sessions and collated, and exercise may cause red spots, redness, pain, and discomfort in the upper limbs during or after exercise sessions. These symptoms will not be considered adverse effects. There may be discomfort due to blood collection; however, in order to minimize discomfort, an experienced nurse will perform the collection. We define adverse events as cases in which dizziness occurs, tingling/paresthesia in the upper limb, bruising, during or after training. If one of the volunteers has these symptoms, the training session will be stopped immediately. 
Frame 1. SPIRIT Study design schedule.

\begin{tabular}{|c|c|c|c|c|c|}
\hline \multirow[b]{2}{*}{ TIME POINT } & \multirow{2}{*}{$\begin{array}{l}\text { Screening } \\
\text { Week- } 2\end{array}$} & \multirow{2}{*}{$\begin{array}{l}\text { Baseline } \\
\text { Week } 0\end{array}$} & \multicolumn{2}{|c|}{$\begin{array}{l}\text { Eight weeks } \\
\text { (intervention period) }\end{array}$} & \multirow{2}{*}{$\begin{array}{l}\begin{array}{l}\text { Four weeks } \\
\text { (detraining } \\
\text { period) }\end{array} \\
12 \text { week }\end{array}$} \\
\hline & & & $\begin{array}{l}\text { After / before the } \\
\text { first session }\end{array}$ & $\begin{array}{l}\text { Four and } \\
\text { eight weeks }\end{array}$ & \\
\hline \multicolumn{6}{|l|}{ ENROLMENT } \\
\hline Eligibility screen & $\mathrm{X}$ & & & & \\
\hline Informed consent & $\mathrm{X}$ & & & & \\
\hline Allocation & & $\mathrm{X}$ & & & \\
\hline \multicolumn{6}{|l|}{ INTERVENTIONS } \\
\hline $\mathrm{PBM} / \mathrm{sMF}+\mathrm{BFR}$ & & & \multicolumn{2}{|c|}{$\longleftrightarrow$} & \\
\hline Placebo + BFR & & & \multirow{2}{*}{\multicolumn{2}{|c|}{$\stackrel{\longrightarrow}{\longrightarrow}$}} & \\
\hline $\mathrm{PBM} / \mathrm{sMF}+\mathrm{HLT}$ & & & & & \\
\hline Placebo + HLT & & & \multicolumn{2}{|c|}{$\stackrel{\longrightarrow}{\longrightarrow}$} & \\
\hline \multicolumn{6}{|l|}{ ASSESSMENT } \\
\hline $\begin{array}{ll}\text { Maximal } & \text { voluntary } \\
\text { contraction } & \end{array}$ & & $\mathrm{X}$ & & $\mathrm{X}$ & $\mathrm{X}$ \\
\hline Resistance to fatigue & & $\mathrm{X}$ & & $X$ & $\mathrm{X}$ \\
\hline Arm circumference & & $\mathrm{X}$ & & $\mathrm{X}$ & $\mathrm{X}$ \\
\hline Oxidative Stress & & & $\mathrm{X}$ & $\mathrm{X}$ & $\mathrm{X}$ \\
\hline Muscle Damage & & & $\mathrm{X}$ & $\mathrm{X}$ & $\mathrm{X}$ \\
\hline Inflammatory levels & & & $\mathrm{X}$ & $\mathrm{X}$ & $\mathrm{X}$ \\
\hline
\end{tabular}

Note: BFR, blood flow restriction, PBMT/sMF, photobiomodulation therapy, HLT, High Load Training.

\section{Warm-up}

In short, voluntaries will then perform a general warm-up on a cycle ergometer (Acte Sports, São Paulo, Brazil) for 5min without load in the position seating.

\section{Photobiomodulation therapy with static magnetic field (PBMT/sMF)}

The physical therapist responsible for the application of PBMT/sMF will determine the groups' experimental and placebo. PBMT/sMF will be used pre-exercise in all sessions, the emitter equipment used was the portable model MR5-ACTIVET PRO, Multi Radiance Medical ${ }^{\circledR}$ (Solon, OH, USA), shown in Table 1. The cluster will be applied in direct contact with the skin stationary at four points of the upper limb (biceps brachii), according to Leal Junior et $\mathrm{al}^{(32)}$, as demonstrated in Figure 2.

The voluntaries will be used a blindfold so that they don't identify whether the application will be placebo or effective since the device does not produce any thermal sensation on the patient's skin and sounds will be emitted independently from the application form. This researcher will not have knowledge about the allocation of BFR or HLT groups.

\section{Blood Flow Restriction training (BFR)}

The groups that will be submitted by BFR training, during the training sessions, voluntaries will be wearing a specially designed elastic pressure cuff ( $33 \mathrm{~mm}$ in width, KAATSU Air Band) around the most proximal portion of the upper arm. In the first and second weeks of training will be used the initial pressure $120 \mathrm{mmHg}$ during BFR session, the cuff will insufflate through KAATSU Nano device (Sato Sports Plaza, Tokyo, Japan). The pressure will be increased by 10 $\mathrm{mmHg}$ in the third-week training session, and this pressure will be worn until the end of the fourth week. The same protocol will be used starting the fifth week, conform shown in Table 2.

The BFR groups will use a load of $30 \%$ de MVC to realize the protocol exercise. Immediately after the exercise bout, the pressure cuff will be quickly removed. The expert, certificated and specialist physical therapist in BFR training will conduct the session. This researcher will be blind as to the application of PBM/ sMF.

\section{Exercise protocol}

The voluntaries will perform the Scott seat sedation exercise with their knees and hips flexed at $90^{\circ}$. The training will be held twice a week for 
Table 1. Parameters for Activ Pro® device.

\begin{tabular}{|c|c|}
\hline Class & $1 \mathrm{M}$ \\
\hline Number of lasers & $\begin{array}{l}\text { 1 Super-pulsed } \\
\text { infrared }\end{array}$ \\
\hline Wavelength (nm) & $905( \pm 1)$ \\
\hline Frequency $(\mathrm{Hz})$ & 250 \\
\hline Peak power $(\mathrm{W})$ & 50 \\
\hline Average mean optical output (mW) & 1.25 \\
\hline Power density $\left(\mathrm{mW} / \mathrm{cm}^{2}\right)$ & 2.84 \\
\hline Energy density $\left(\mathrm{J} / \mathrm{cm}^{2}\right)$ & 0.085 \\
\hline Dose $(J)$ & 0.0375 \\
\hline Spot size of laser $\left(\mathrm{cm}^{2}\right)$ & 0.44 \\
\hline Number of red LEDs & 3 Red \\
\hline Wavelength of red LEDs (nm) & $640( \pm 10)$ \\
\hline Frequency $(\mathrm{Hz})$ & 2 \\
\hline Average optical output $(\mathrm{mW})$ - each & 66.67 \\
\hline Power density $\left(\mathrm{mW} / \mathrm{cm}^{2}\right)-$ each & 74.08 \\
\hline Energy density $\left(\mathrm{J} / \mathrm{cm}^{2}\right)-$ each & 2.22 \\
\hline Dose $(\mathbf{J})$ - each & 2.00 \\
\hline Spot size of red LED $\left(\mathrm{cm}^{2}\right)-$ each & 0.9 \\
\hline Number of infrared LEDs & 3 Infrared \\
\hline Wavelength of infrared LEDs (nm) & $875( \pm 10)$ \\
\hline Frequency $(\mathrm{Hz})$ & 16 \\
\hline Average optical output $(\mathrm{mW})$ - each & 83.33 \\
\hline Power density $\left(\mathrm{mW} / \mathrm{cm}^{2}\right)-$ each & 92.59 \\
\hline Energy density $\left(\mathrm{J} / \mathrm{cm}^{2}\right)$ - each & 2.77 \\
\hline Dose $(\mathrm{J})$ - each & 2.50 \\
\hline Spot Size of LED $\left(\mathrm{cm}^{2}\right)-$ each & 0.9 \\
\hline Magnetic Field (mT) & 35 \\
\hline Irradiation time per site ( $\mathrm{sec}$ ) & 60 \\
\hline Total energy irradiated per site $(\mathrm{J})$ & 13.5375 \\
\hline $\begin{array}{l}\text { Number of sites irradiated per } \\
\text { treatment }\end{array}$ & 4 \\
\hline $\begin{array}{l}\text { Total energy irradiated per treatment } \\
(\mathrm{J})\end{array}$ & 54.15 \\
\hline Aperture of the device $\left(\mathrm{cm}^{2}\right)$ & 4 \\
\hline Application mode & $\begin{array}{l}\text { Cluster probe held } \\
\text { stationary in skin } \\
\text { contact with a 90- } \\
\text { degree angle and } \\
\text { slight pressure }\end{array}$ \\
\hline
\end{tabular}

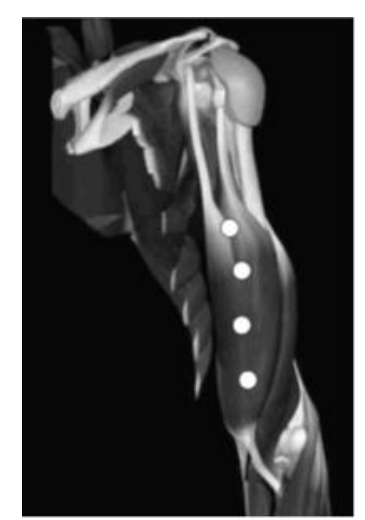

Figure 2. Point of application PBMT/sMF
Table 2. Cuff pressures during protocol exercise

\begin{tabular}{ll}
\hline Cuff Pressure & (Week.Session) \\
\hline $120 \mathrm{mmHg}$ & $1.1-1.2-2.1-2.2$ \\
$130 \mathrm{mmHg}$ & $3.1-3.2-4.1-4.2$ \\
$120 \mathrm{mmHg}$ & $5.1-5.2-6.1-6.2$ \\
$130 \mathrm{mmHg}$ & $7.1-7.2-8.1-8.2$ \\
\hline
\end{tabular}

eight weeks, totaling 16 sessions. The exercise that will be proposed is Scott elbow flexion unilaterally, with a 30\% MVC pre-assessment load for BFR groups and $80 \%$ MVC for HLT groups. During the fourth week the load will be adjusted, and thus the value of $30 \%$ of the MVC of the assessment that occurred at the end of the fourth week will be used.

The BFR protocol will consist of four sets of 20 repetitions or until failure with a rest interval of 30 seconds between sets, being stimulated with the standard verbal command for all voluntaries in BFR groups. For to HLT group the participants will be submitted three sets with 10 repetitions. Rest periods between set will be one minute. The execution speed in BFR and HLT groups will be controlled by a metronome set at three seconds, which was equally divided between concentric and eccentric muscle action. This process will be realized for a physical therapy and the duration of each session of exercise will be between 5 to 6 minutes.

\section{Outcomes Measures \\ Maximum Voluntary Contraction (MVC) and Muscle Fatigue Induction Protocol (MFIP)}

The protocol for determining muscle strength used the maximum voluntary contraction (MVC) capacity and endurance will be followed by a blind researcher (a trained physiotherapist of Sports Medicine Institute) and will occur on the Biodex 4 Pro System isokinetic dynamometer (Biodex Medical Systems, USA). The positioning of the equipment in the equipment will be performed according to the manufacturer's instructions for the evaluation of the forearm flexion movements. As the tests will be performed unilaterally (non-dominant arm) and the positioning of the equipment will remain the same in all evolutions for each volunteer.

The first part of the isokinetic dynamometer protocol is to determine the maximum isometric torque of the elbow flexors (biceps brachii). To this end, three voluntary contractions (VC) will be performed in the isometric mode at the $45^{\circ}$ elbow flexion position, lasting $5 s$ and a $5 s$ interval between contractions. During VCs, a constant and standardized verbal stimulus will be performed by the researchers. The highest isometric torque value achieved in the VCs will be considered the 
maximum voluntary contraction (MVC). After the determination of MVC, a period of 180 seconds will be respected before the initiation of the muscle fatigue induction protocol (MFIP) to verify the resistance to fatigue.

The MFIP will consist of a set of 20 concentric/eccentric elbow flexor contractions. Contractions will be performed at $90^{\circ}$ amplitude and $90^{\circ}$.sec -1 velocity for eccentric contractions and $60{ }^{\circ}$.sec - 1 for concentric contractions. The guidance provided to the volunteers was to employ the highest strength possible to execute the elbow flexion movement and resist the elbow extension movement imposed by the dynamometer from the first to the last repetition.

\section{Arm circumference}

A researcher (physical therapy) previously trained and blinded to the experimental and placebos groups will evaluate arm circumference as follows, with free upper limb thus discarding the possibility that garments will increase and influence the result. With the patient in a sitting position, the researcher will ask the volunteer to move away from the upper limb of the trunk and thus will circumvent the upper limb in five, ten and 15 centimeters above the reference point (lateral epicondyle of the humerus), with the tape measure to evaluate the circumference of this limb.

\section{Blood samples and biochemical analyses}

A nurse will be responsible to blood collection and won't be informed about groups randomization, blood samples $(10 \mathrm{ml})$ will be collected from the antecubital vein before and 1 minute after the exercise protocol. Blood samples will also be collected 1 minute after the protocol. The samples will be taken by a nurse blinded to the allocation of the volunteers to the four experimental groups. One hour after collection, each sample will be centrifuged at $3600 \mathrm{rpm}$ for 10 minutes. Pipettes will be used to transfer the serum to Eppendorf® tubes (Eppendorf, Hamburg, Germany), which will be stored at $-80^{\circ} \mathrm{C}$ until analysis.

Blood analysis will involve the determination of CK activity as indirect marker of muscle damage, using spectrophotometry and specific reagent kits (Labtest Diagnóstica, Lagoa Santa, MG, Brazil); IL6 and TNF-a levels as inflammatory markers, using ELISA and specific reagents (R\&D Systems, Minneapolis, MN, USA); and thiobarbituric acid reactive substances (TBARS), carbonylated proteins, catalase (CAT), superoxide dismutase (SOD), and total antioxidant capacity (TAC) as markers of oxidative stress, spectrophotometry and specific reactions.

using

\section{Data Analysis \\ Calculation of sample size}

For sample calculation, the value of $\beta$ will be considered to be $20 \%$ and $\alpha$ of $5 \%$. The total number of voluntaries in the survey was calculated based on data presented by De Marchi et al (33) The author conducted a study to analyses the effects of PBMT on the performance of futsal players and recovery in an uncontrolled field environment, taking into account the effect size of 0.65 found in the results obtained in this study Using these data for sample calculation (using Gpower 3.1), we obtained as a result $n$ from 44 volunteers (final sample, excluding possible losses).

\section{Statistical Analysis}

The statistical analysis it will be conducted following intention-to-treat principles (i.e., the voluntaries it will be analyzed in the groups to which they were allocated). Data normality it will be tested by Kolmogorov-Smirnov test. If the data shows normal distribution, two-way repeated measures analysis of variance with post hoc Bonferroni correction will be realized. If the data do not show a normal distribution (non-parametric) will be analyzed using the Friedman test and, secondarily, the Wilcoxon signed-rank test. The association between categorical variables will be analyzed using the Chi-square test or Fisher's exact test Data will be expressed as mean and standard deviation, mean difference between treatments, and 95\% confidence intervals $(95 \% \mathrm{Cls})$. Data will be also expressed as frequency (\%). The significance level was set at $p<0.05$. The magnitude of differences (Cohen-d) between groups, to examine practical significances, was calculated using the mean and SD of placebo, PBMT-sMF and BFR treatments (using Gpower 3.1). We adopted the criteria of Cohen for the analysis (0.2: small; 0.50: moderate; 0.80 : large). All analyses will be calculated one of the researchers who be not involved in data collection.

\section{DISCUSSION}

In the proposed study, the effects of the BFR associated with PBMT/SMF will be identified and compared with the effects of BFR training, HLT, both in separated form and associated with previous application of PBMT/sMF on muscle performance (strength fatigue resistance). Moreover, the acute (one session) and long-term 
effects (eight weeks) of the training protocol on markers inflammation, oxidative stress, muscle damage will be verified to determine the changes occurred in each intervention.

This will be the first study to evaluate the acute, long-term effects of BFR and PBMT / sMF isolated and combined on indirect exerciseinduced oxidative stress. It is currently known that production of reactive oxygen species (ROS) plays a vital role in cell function in terms of acting as a signaling molecule ${ }^{(34)}$. In addition, ROS play an important role in regulating muscle strength production and adaptive responses induced by exercise training ${ }^{(34,35)}$. On the other hand, a systemic overproduction of ROS in skeletal muscle promotes an interruption of signaling and redox control, which can damage cellular components (lipids, proteins and DNA), cell dysfunction and even cause apoptosis ${ }^{(34)}$.

Furthermore, the increase in ROS can elevate the levels of pro-inflammatory cytokines and, consequently, cause contractile dysfunction, fatigue and decreased performance. The implication of this redox imbalance is a longer period of muscle recovery, therefore, impairing the quality of training sessions and exerciseinduced adaptive response. ${ }^{(36)}$ This is a fundamental issue, especially for subjects exposed to periods of intense training and in the musculoskeletal rehabilitation process. Thus, this study has a relevant impact on clinical practice, as it will demonstrate whether the application of PBMT/sMF with BFR can be feasible for healthy and unhealthy patients, as well as elucidating the possible mechanisms responsible for its effects.

In addition, we will investigate the effects of a period of physical activity interruption, that is, a period of detraining. As strengths of this study, we can highlight that it has high methodological quality because it is randomized and prospectively registered, masks the evaluators and patients, conceals allocation, and uses an intention-to-treat approach. We also calculated sample size in order to provide adequate statistical power to identify possible differences in the main outcomes.

The limitations of study are the use the circumference of arm to evaluation the muscle volume trough brachii biceps and not magnetic resonance image. However, in clinical practice, the circumference of arm is the most used method for assessing muscle volume due to its costbenefit and easy application, presenting quantitative results ${ }^{(37)}$. The selection criteria of the study exclude women and the voluntaries are only male, this occurred so that there was no influence of the woman's menstrual cycle; once there was biochemical analysis of biomarkers of muscle damage and oxidative stress. The results from this study will be disseminated through scientific publications in international peerreviewed journals and presentations at national and international scientific meetings.

Authors' contributions: All cited authors participated in these steps. JVF: Conceptualization, Methodology, Project Administration, Visualization, Writing. TDM: Conceptualization, Methodology, Formal Analysis, Validation, Supervision, Writing. ECPLJ and MS: Validation, Supervision, Writing. All authors have read and approved the final version of the manuscript.

Conflict of interest: Professor Ernesto Cesar Pinto Leal-Junior receives research support from Multi Radiance Medical (Solon, $\mathrm{OH}$ - USA), a laser device manufacturer. Multi Radiance Medical had no role in the planning of this study. They had no influence on study design, data collection and analysis, decision to publish, or preparation of the manuscript. The remaining authors declare that they have no conflict of interest.

Transparency statement: The JVF affirms that this manuscript is an honest, accurate, and transparent account of the study being reported; that no important aspects of the study have been omitted; and that any discrepancies from the study as planned (and, if relevant, registered) have been explained".

\section{REFERENCES}

1. Shaw I, Shaw BS, Brown GA, Shariat A. Review of the Role of Resistance Training and Musculoskeletal Injury Prevention and Rehabilitation. J Orthop Res Ther. 2016; 102:1-5.

2. Bea JW, Blew RM, Howe C, HetheringtonRauth M, Going SB. Resistance Training Effects on Metabolic Function Among Youth: A Systematic Review. Pediatr Exerc Sci. 2017; 29 (3): 297-315.

3. Chan DC, Chang CB, Han DS, et al. Effects of exercise improves muscle strength and fat mass in patients with high fracture risk: $A$ randomized control trial. J Formos Med Assoc. 2018; 117(7): 572-582.

4. Lesinski M, Prieske O, Granacher U. Effects and dose-response relationships of resistance training on physical performance in youth athletes: a systematic review and meta-analysis. $\mathrm{Br} \mathrm{J}$ Sports Med. 2016; 50(13): 781-95.

5. American College of Sports Medicine. American College of Sports Medicine position stand. Progression models in resistance training for healthy adults. Med Sci Sports Exerc. 2009; 41(3):687-708.

6. Kraemer WJ, Ratamess NA. Fundamentals of resistance training: progression and 
exercise prescription. Med Sci Sports Exerc. 2004; 36: 674-688.

7. Sato $Y$. The history and future of KAATSU Traning. Int J Kaatsu Train Res. 2005; 1:1-5.

8. Abe T, Fujita S, Nakajima T, et al. Effects of Low-Intensity Cycle Training with Restricted Leg Blood Flow on Thigh Muscle Volume and VO2MAX in Young Men. J Sports Sci Med. 2010; 9(3): 452-458.

9. Laurentino GC, Ugrinowitsch C, Roschel, H, et al. Strength training with blood flow restriction diminishes myostatin gene expression. Med Sci Sports Exerc. 2012; 44: 406-412.

10. Karabulut M, Abe T, Sato, Y, Bemben MG. The effects of low-intensity resistance training with vascular restriction on leg muscle strength in older men. Eur J Appl Physiol. 2010;108: 147-155.

11. Cardoso RK, Araujo AM, Freitas MP, Rombaldi AJ. Effect of training with partial blood flow restriction in older adults: a systematic review. Rev Bras Cineantropom Hum. 2018; 20(2):219-228.

12. Ferlito JV, Pecce SAP, Oselame L, De Marchi $\mathrm{T}$. The blood flow restriction training effect in knee osteoarthritis people: a systematic review and meta-analysis Clin Rehabil. 2020;34(11):1378-1390. doi:10.1177/0269215520943650

13. Takarada $Y$, Nakamura $Y$, Aruga S, Onda T , Miyazaki S, Ishii, N. Rapid increase in plasma growth hormone after low-intensity resistance exercise with vascular occlusion. J Appl Physiol. 2000; 88:61-65.

14. Takarada, $Y$, Sato $Y$, Ishii N. Effects of resistance exercise combined with vascular occlusion on muscle function in athletes. Eur J Appl Physiol. 2002; 86: 308-314.

15. Ivey FM., Roth SM, Ferrell RE, et al. Effects of age, gender, and myostatin genotype on the hypertrophic response to heavy resistance strength training. J Gerontol A Biol Sci Med Sci. 2000; 55(11): M641-648.

16. Enoka RM, Stuart DG. Neurobiology of muscle fatigue. J Appl Physiol. 1992; 72: 1631-1648.

17. Allen DG, Lamb GD, Westerblad H. Skeletal muscle fatigue: cellular mechanisms. Physiol Rev. 2008; 88(1):287-332.

18. Cheung, K., Hume, P. \& Maxwell, L. Delayed onset muscle soreness: treatment strategies and performance factors. Sports Med. 2003; 33 (2): 145-64.
19. Kjeldsen SS, Næss-Schmidt ET, Hansen GM, Nielsen JF, Stubbs PW. Neuromuscular effects of dorsiflexor training with and without blood flow restriction. Heliyon. 2019; 5 (8): e02341.

20. Rossi FE, de Freitas MC, Zanchi NE, Lira FS, Cholewa JM. The Role of Inflammation and Immune Cells in Blood Flow Restriction Training Adaptation: A Review. Front Physiol. 2018;9:1376.

21. Powers SK, Bomkamp M, Ozdemir M, Hyatt $\mathrm{H}$. Mechanisms of exercise-induced preconditioning in skeletal muscles. Redox Biol. 2020;35:101462.

22. Vanin AA, Miranda EF, Machado CS, et al. What is the best moment to apply phototherapy when associated to a strength training program? A randomized, doubleblinded, placebo-controlled trial : Phototherapy in association to strength training [published correction appears in Lasers Med Sci. 2017 Jan;32(1):253]. Lasers Med Sci. 2016;31(8):1555-1564

23. Leal-Junior ECP, Lopes-Martins RÁB, Bjordal JM. Clinical and scientific recommendations for the use of photobiomodulation therapy in exercise performance enhancement and postexercise recovery: current evidence and future directions. Braz J Phys Ther. 2019;23(1):71-75.

24. De Marchi T, Leal Junior EC, Bortoli C, Tomazoni SS, Lopes-Martins RA Salvador M. Low-level laser Therapy (LLLT) in human progressive-intensity running: effects on exercise performance, skeletal muscle status, and oxidative stress. Lasers Med Sci. 2012; 27(1): 231-236.

25. De Marchi T, Leal-Junior ECP, Lando KC, Cimadon F, Vanin AA, da Rosa DP, Salvador M. Photobiomodulation therapy before futsal matches improves the staying time of athletes in the court and accelerates post-exercise recovery. Lasers Med Sci. 2019; 34(1):139-148.

26. Ferlito JV, Ferlito MV, Leal-Junior ECP, Tomazoni SS, De Marchi T. Comparison between cryotherapy and photobiomodulation in muscle recovery: a systematic review and meta-analysis [published online ahead of print, 2021 Oct 20]. Lasers Med Sci. 2021;10.1007/s10103021-03442-7. 
27. Vanin AA, Verhagen E., Barboza SD, Costa LOP, Leal-Junior ECP. Photobiomodulation therapy for the improvement of muscular performance and reduction of muscular fatigue associated with exercise in healthy people: a systematic review and metaanalysis. Lasers Med Sci. 2018; 33(1): 181214.

28. de Paiva PRV, Casalechi HL, Tomazoni SS, et al. Does the combination of photobiomodulation therapy (PBMT) and static magnetic fields (sMF) potentiate the effects of aerobic endurance training and decrease the loss of performance during detraining? A randomised, triple-blinded, placebo-controlled trial. BMC Sports Sci Med Rehabil 2020;12:23.

29. De Marchi T, Frâncio F, Ferlito JV, et al. Effects of Photobiomodulation Therapy Combined with Static Magnetic Field in Severe COVID-19 Patients Requiring Intubation: A Pragmatic Randomized Placebo-Controlled Trial. J Inflamm Res. 2021;14:3569-3585.

30. Chan A-W, Tetzlaff JM, Altman DG, Laupacis A, Gøtzsche PC, Krleža-Jerić K, et al. SPIRIT 2013 Statement: Defining Standard Protocol Items for Clinical Trials. Ann Intern Med. 2013;158:200.

31. Yamato T, Maher C, Saragiotto B, Moseley A, Hoffmann T, Elkins M, et al. The TIDieR checklist will benefit the physical therapy profession. Braz J Phys Ther. 2016;20:1913

32. Leal Junior EC, Lopes-Martins RA, Vanin $\mathrm{AA}$, et al. Effect of $830 \mathrm{~nm}$ low-level laser therapy in exercise-induced skeletal muscle fatigue in humans. Lasers Med Sci. 2009;24(3):425-431.

33. De Marchi T, Schmitt VM, Machado GP, et al. Does photobiomodulation therapy is better than cryotherapy in muscle recovery after a high-intensity exercise? A randomized, double-blind, placebocontrolled clinical trial. Lasers Med Sci. 2017;32(2):429-437

34. Radak Z, Zhao Z, Koltai E, Ohno H, Atalay $M$. Oxygen consumption and usage during physical exercise: the balance between oxidative stress and ROS-dependent adaptive signaling. Antioxid Redox Signal. 2013;18(10):1208-1246.

35. Spanidis Y, Veskoukis AS, Papanikolaou C, et al. Exercise-Induced Reductive Stress Is a Protective Mechanism against Oxidative Stress in Peripheral Blood Mononuclear
Cells. Oxid Med Cell Longev. 2018;2018:3053704.

36. Bessa AL, Oliveira VN, Agostini GG, et al. Exercise Intensity and Recovery: Biomarkers of Injury, Inflammation, and Oxidative Stress. J Strength Cond Res. 2016;30(2):311-319

37. Rodrigues Neto G, Silva JCG da, Freitas L, Silva HG da, Caldas D, Novaes $J$ da $S$, Cirilo-Sousa MS. Effects of strength training with continuous or intermittent blood flow restriction on the hypertrophy, muscular strength and endurance of men. Acta Sci. Health Sci;41(1):e42273. 